information. Black Urjanis usually breed true ; when they fail to do so, the progeny is generally a sport of a particular kind called dijji, uniformly red when young, more or less mottled with white when adult. These dijjis are apt to "throw back," and in turn produce good Urjanis.

One of my own mottled birds (dijji) remains in my possession, and is now mated to an Urjani-an own brother. The pair has produced this spring four young : three dijiis, just like the mother, and one partial reversion to the Urjani form, being dark-chequered blue, with red bars on the wings. The original parent pair of Urjanis have also raised four squabs this season - three normal and one dijji; sexes not yet determined. I state these facts without comment; but would be glad to know whether fanciers in England or elsewhere have observed anything quite as striking in the way of colour-variation.

Beyrout, Syria.

W. T. VAN DYCK.

\section{Dependence of the Colour of Solutions on the Nature of the Solvent.}

Ir is a well-known fact that the colour exhibited by one and the same body in solution depends more or less on the nature of the solvent. In some cases this phenomenon can be satisfactorily accounted for by electrolytic dissociation, but in the majority of cases hitherto examined this explanation is not admissible. Perhaps the most striking of these is that of iodine, the solutions of which are coloured variously violet, blue, brown, and yellow. The hypothesis has been put forward that the variation in absorption might be due to the formation of molecular aggregates of variable complexity; but this, at least in the case of iodine, has been rendered very improbable by the recent researches of Beckmann and others. Nor does the hypothesis that the variation may be due to a varying degree of combination with the solvent seem much more promising.

If, now, absorption be a case of electrical resonance, should one not expect a relation between the absorption of the dissolved body and the physical properties of the solvent, sufficient to account for the observed variations? That such a relation should exist, seems possible from the following rough considerations.

The period of vibration of an electric oscillator is, in the usual notation,

$$
\mathrm{T}=2 \pi \sqrt{\mathrm{LC}}
$$

where $\mathrm{L}=$ self-induction, and $\mathrm{C}=$ capacity. But now :-

$$
\mathrm{LC}=g \mathrm{~K} \mu,
$$

where $g$ is a geometrical factor and $\mathrm{K}$ and $\mu$ are the dielectric constant and permeability of the surrounding medium. Also $n^{2}=\mathrm{K} \mu$, where $n$ is the index of refraction of the medium for very long waves, whence it follows that

$$
\mathrm{T}=2 \pi n \sqrt{g},
$$

which means that the principal absorption-band should travel towards the red end of the spectrum as the index of refraction of the solvent increases. This result is identical with the general qualitative law enunciated many years ago by Kundt, on the basis of experimental data. There are, it is true, various breaks in the parallelism ; still this mode of viewing the question seems to offer more possibilities than the others.

Holywood, Belfast.

F. G. Donnan.

\section{Hatching Lizards' Eggs.}

CAN any of your readers suggest a way to hatch lizards' eggs? I have had a pair of bright-green lizards ( $I$ think they came from Italy) in a glass vivarium in a very sunny window for two years and a half. Last year, on May I9, the female laid eleven eggs. I left them exactly as the mother laid them, and after about three weeks I opened one and found the rudiments of a young lizard; but the other eggs never came to anything. I should like to rear them this year if it is possible.

H. A. Ross.

Trevean, Penzance.

\section{THE DIFFUSION OF METALS.}

$\mathrm{T}^{\mathrm{T}}$ is now quite usual to think of alloys as being solid solutions and to recogrise that the atoms of solid metals are in active movement. That this must be the case, is revealed by the passage of metals to allotropic NO. I 386 , vOL. 54] modifications in which the physical properties differ widely from those of the same metals in their normal state. It is well, th erefore, that we should remember how much was done for us thirty years ago by Matthiessen in framing such views, and by Graham in showing that solid metals are true solvents for gases which move and diffuse freely in them, sometimes to reappear with gaseous elasticity.

The experimental portion of the latter work, Graham entrusted to me, and my hope that I should be able to extend his work on the diffusion of salts, to liquid and solid metals, has been somewhat tardily realised by the delivery in the present year of the "Bakerian Lecture" of the Royal Society, of which the following is a brief abstract.

PART I.-Diffusion of Molten Metals.

In the first part of it allusion is made to some earlier experiments of my own conducted in 1883 on the diffusion of gold, silver, and platinum in molten lead. It is.strange that although the action of osmotic pressure in lowering the freezing point of metals has been carefully examined, very little attention has been devoted to the measurement, or even to the consideration, of the molecular movements which enable two or more metals to form a truly homogeneous fluid mass. The absence of direct experiments on the diffusion of molten metals is probably explained by the want of a sufficiently accurate method. Ostwald has stated, moreover, with reference to the diffusion of salts, that "to make accurate experiments in diffusion is one of the most difficult problems in practical physics," and the difficulties are obviously increased when molten metals diffusing into each other take the place of salts diffusing into water

The continuation of the research was mainly due to the interest Lord Kelvin had always taken in the experiments. The want of a ready method for the measurement of comparatively high temperatures, which led to the abandonment of the earlier work, was overcome when the recording pyrometer was devised, and the use of thermo-junctions in connection with this instrument rendered it possible to measure and record the temperature at which diffusion occurred. Thermo-junctions were placed in three or more positions in either a bath of fluid metal or an oven carefully kept hotter at the top than at the bottom. In the bath or oven, tubes filled with lead were placed, and in this lead, gold, or a rich alloy of gold, or of the metal under examination, was allowed to diffuse upwards against gravity. The amount of metal diffusing in a given time was ascertained by allowing the lead in the tubes to solidify; the solid metal was then cut into sections, and the amount of metal in the respective sections determined by analysis.

The movement in linear diffusion is expressed, in accordance with Fick's law, by the differential equation

$$
\frac{d v}{d t}=k \frac{d^{2} v}{d x^{2}} \text {. }
$$

In this equation $x$ represents distance in the direction in which diffusion takes place, $v$ is the degree of concentration of the diffusing metal, and $t$ is the time ; $k$ is the diffusion constant, that is, the number which expresses the quantity of the metal in grams diffusing through unit area (I sq. cm.) in unit time (one day) when unit difference of concentration (in grams per c.c.) is maintained between the two sides of a layer I cm. thick. The experiments described in the Bakerian Lecture showed that metals diffuse in one another just as salts do in water, and the results were ultimately calculated by the aid of tables prepared by Stefan for the calculation of Graham's experiments on the diffusion of salts, special tables being calculated by one of my students, Mr. A. Stansfield, in connection with this research.

The necessary precautions to be observed and the corrections to be made were described at length and the 